\title{
Hepatocyte RXRalpha deficiency in matured and aged mice: impact on the expression of cancer-related hepatic genes in a gender-specific manner
} Minglei Guo' ${ }^{1}$ Lei Gong², Lin Heㄹ, Lois Lehman-McKeeman² and YuJui Yvonne Wan*1

\author{
Address: ${ }^{1}$ Department of Pharmacology, Toxicology \& Therapeutics, University of Kansas Medical Center, Kansas City, KS, 66103, USA and \\ ${ }^{2}$ Discovery Toxicology, Bristol-Myers Squibb Company, Princeton, NJ 08543, USA \\ Email: Minglei Guo - mguo@kumc.edu; Lei Gong - lei.gong@bms.com; Lin He - lhe@kumc.edu; Lois Lehman-McKeeman - Lois.Lehman- \\ McKeeman@bms.com; Yu-Jui Yvonne Wan* - ywan@kumc.edu \\ * Corresponding author
}

Published: 28 August 2008

BMC Genomics 2008, 9:403 doi:10.1186/147I-2164-9-403

This article is available from: http://www.biomedcentral.com/I47I-2/64/9/403

(C) 2008 Guo et al; licensee BioMed Central Ltd.

This is an Open Access article distributed under the terms of the Creative Commons Attribution License (http://creativecommons.org/licenses/by/2.0), which permits unrestricted use, distribution, and reproduction in any medium, provided the original work is properly cited.

\begin{abstract}
Background: The occurrence of liver cancer is higher in males than in females, and the incidence increases during aging. Signaling pathways regulated by retinoid $\times$ receptor $\alpha(R \times R \alpha)$ are involved in hepatocellular carcinogenesis. The phenotype of hepatocyte $R X R \alpha$ deficient mice is different between genders. To explore the impact of hepatocyte $R X R \alpha$ deficiency on gender-dependent hepatic gene expression, we compared the expression profiles of cancer-related genes in 6 and 24 month old male and female mice.

Results: In 6 month old mice, male mutant mice showed more cancer-related genes with alteration in mRNA levels than females did (195 vs. 60). In aged mice ( 24 month), female mutant mice showed greater deviation in mRNA expression levels of cancer-related genes than their male counterparts (149 vs. 82). The genes were classified into five categories according to their role in carcinogenesis: apoptosis, metastasis, cell growth, stress, and immune respnse. In each category, dependent upon age and gender, the genes as well as the number of genes with altered mRNA levels due to $R X R \alpha$ deficiency varies.

Conclusion: The change in hepatic cancer-related gene expression profiles due to $R X R \alpha$ deficiency was gender- and age-dependent. The alteration of mRNA levels of cancer-related genes implied that aberrant $R X R \alpha$ signaling could potentially increase the risk of liver cancer and that retinoid signaling might contribute to gender- and age-associated liver cancer incidence.
\end{abstract}

\section{Background}

RXRs (Retinoid $\times$ Receptors), belonging to the nuclear receptor superfamily, play important roles in detoxification, apoptosis, differentiation, and proliferation through hetero-dimerizing with other nuclear receptors [1]. RXR $\alpha$, $\beta$, and $\gamma$ are the receptors for retinoids, and have been used to prevent and treat cancer. RXR $\alpha$ is the most prevalent receptor expressed in liver. Aberrant RXR $\alpha$-induced pathways have been implicated as possible mechanisms for the development of hepatocellular carcinoma $[2,3]$. Hepatocyte-specific RXR $\alpha$-deficient mice were first generated by Wan etc. $[4,5]$. Although hepatocyte RXR $\alpha$ defi- 
ciency does not show an obvious phenotype, many metabolic pathways including fatty acid, cholesterol, and xenobiotic are compromised due to hepatocyte RXR $\alpha$ deficiency. Furthermore, shortened hepatocyte lifespan and impaired capacity for liver regeneration after partial hepatectomy are detected in hepatocytes that do not express RXR $\alpha$ [6]. These findings indicate that hepatocyte RXR $\alpha$ is not only important for liver metabolism, but also in control of hepatocyte proliferation and survival.

The impact of RXR $\alpha$ deficiency on the expression of RXR $\alpha$ target genes is gender dependent. The expression of cytochrome P450 (CYP450) genes including Cyp4a, 3a, and $2 \mathrm{~b}$ are differentially expressed in male and female hepatocyte RXR $\alpha$-deficient mice [7]. Using sex hormone treatments, we have previously shown that male hormones might have an impact on regulating RXR $\alpha$-mediated signaling [7]. In addition to gender, aging also imposes significant changes on nuclear receptor-mediated gene expression in hepatocytes [8]. Nuclear receptor signaling pathways are in hypo-functioning status in an aged person's peripheral blood mononuclear cells [9]. The incidence of liver cancer is much higher in males than in females, and increases with aging. Based on these observations, we hypothesize that aberrant hepatocyte RXR $\alpha$ signaling might have complex repercussions on cell biological activities and contribute to the risk of liver carcinogenesis in an age and gender dependent manner.

To study the impact of hepatoctye RXR $\alpha$ deficiency on cancer-related gene expression in each gender, we have performed microarray analyses using livers derived from 6 and 24 month old male and female wild type and hepatocyte RXR $\alpha$-deficient mice. It is generally recognized that 6 month old mice are mature and 24 month old mice are aged $[10,11]$. We used Ingenuity Pathway software to identify cancer-related genes. Generally, these genes can be classified into five categories that are associated with carcinogenesis: 1) apoptosis; 2) stress response; 3) cell migration; 4) cell cycle/growth regulation; and 5) immune response. Our data demonstrated that in 6 month old mice, hepatocyte RXR $\alpha$ deficiency resulted in more changes (both in number and fold) of gene expression profiles in male than in female mice; in contrast, in aged mice ( 24 month old), the pattern was reversed with females showing more changes in genetic expression profiles than their male counterparts. Our data provide a database for identification of candidate genes that might account for gender-, age-, and retinoid signaling-associated liver cancer development.

\section{Results and discussion}

In 6 month old hepatocyte RXR $\alpha$ deficient mice, 195 genes found in male mice livers had altered expression patterns while 60 genes had changed expression patterns in female mouse livers. In contrast to the matured mice, in aged mice the number of genes that had altered expression patterns due to hepatocyte RXR $\alpha$ deficiency was higher in female (149) than in male (82) mouse livers (table 1). Our data suggest that hepatocyte RXR $\alpha$ deficiency has a greater impact on males than females in young mice. When mice are aged, the impact is greater in female than in male mice.

To validate the microarray results, we selected 10 genes to quantify mRNA levels by qRT-PCR. All qRT-PCR analyses were performed on the same samples used for the microarray experiments. Table 2 summarized the fold change in mRNA levels detected by qRT-PCR and microarray experiments. Both methods detected similar trends of expression (up or down regulation) although the fold changes may not be the same. There were only a few exceptions i.e. FKBP5 and USP2. The difference in fold change may reflect the sensitivity difference between the two methods. Generally, the results from both methods were consistent.

Many biological processes can be compromised during carcinogenesis. These processes include resistance to apoptosis, unlimited replication potential, self-sufficient growth signal, insensitivity to negative regulators, sustained angiogenesis, and impaired tissue remodeling, all which influence cancer cells to metastasize [12]. In addition, cell-host interactions such as immune response and stress response pathways have been demonstrated to play

Table I: Numbers of cancer related genes with altered mRNA levels.

\begin{tabular}{lcccc}
\hline Comparison & \multicolumn{2}{c}{ 6 month old mice (KO vs. WT) } & 24 month old mice (KO vs. WT) \\
\hline Gender & male & female & male \\
\hline apoptosis & 11 & 6 & 10 & 5 \\
metastasis & 22 & 6 & 9 & 8 \\
stress inducible & 8 & 2 & 4 & 2 \\
cell growth & 39 & 11 & 9 & 22 \\
immune response & 15 & 4 & 10 & 24 \\
unclassified & 100 & 29 & 42 & 61 \\
total & 195 & 60 & 82 & 149 \\
\hline
\end{tabular}


Table 2: Validation the changes of mRNA levels in selected genes.

\begin{tabular}{cccccc}
\hline Comparison & $\begin{array}{c}\text { month old mice } \\
\text { KO vs. WT }\end{array}$ & $\begin{array}{c}24 \text { month old mice } \\
\text { KO vs. WT }\end{array}$ & Methods \\
Gender & male & female & male & female \\
& & & & \\
\hline \multirow{2}{*}{ FKBP5 } & 4.26 & 2.63 & 2.81 & $(-)$ & micro-array \\
& 3.26 & 2.23 & $(-)$ & $(-)$ & qRT-PCR \\
USP2 & 3.46 & 2.17 & 3.97 & $(-)$ & micro-array \\
& 3.14 & 2.2 & $(-)$ & $(-)$ & qRT-PCR \\
Caspase 8 & -2.04 & $(-)$ & -2.31 & $(-)$ & micro-array \\
& -1.98 & $(-)$ & -3.08 & $(-)$ & qRT-PCR \\
THBSI & -7.11 & $(-)$ & $(-)$ & $(-)$ & micro-array \\
& -22.21 & $(-)$ & $(-)$ & $(-)$ & qRT-PCR \\
SERPINEI & -23.48 & $(-)$ & $(-)$ & $(-)$ & micro-array \\
& -11.76 & $(-)$ & $(-)$ & $(-)$ & qRT-PCR \\
FOS & -5.74 & $(-)$ & $(-)$ & $(-)$ & micro-array \\
& -9.94 & $(-)$ & $(-)$ & $(-)$ & qRT-PCR \\
ATF3 & -33.85 & $(-)$ & $(-)$ & $(-)$ & micro-array \\
& -24.32 & $(-)$ & $(-)$ & $(-)$ & qRT-PCR \\
DNAJBI & -11.30 & $(-)$ & $(-)$ & $(-)$ & micro-array \\
& -6.10 & $(-)$ & $(-)$ & $(-)$ & qRT-PCR \\
EGRI & -15.88 & $(-)$ & $(-)$ & $(-)$ & micro-array \\
& -9.33 & $(-)$ & $(-)$ & $(-)$ & qRT-PCR \\
BTG2 & -5.26 & $(-)$ & $(-)$ & $(-)$ & micro-array \\
& -9.34 & $(-)$ & $(-)$ & $(-)$ & qRT-PCR \\
& & & & & \\
\hline
\end{tabular}

(-): No significant change on mRNA expression level.

important roles in carcinogenesis $[13,14]$. To reflect these processes, we classified the cancer-related genes, which changed their expression level due to hepatocyte RXR $\alpha$ deficiency, into the five categories: apoptosis, migration, cell growth regulation, stress induction, and immune response.

\section{Apoptosis-related genes}

In the group of apoptosis-related genes, two general trends were noted in table 3. (1) The number of genes with varied mRNA levels was always higher in male mutant mice than in female mutant mice. In 6 month old mice, 11 apoptosis-associated genes had altered mRNA levels in male mutant mice while in females; only 6 apoptosis genes had changed expression levels. In 24 month old mice, 10 apoptosis-associated genes had significant changes in mRNA levels in male mutant mice; while in females, the number decreased to 5. (2) Most anti-apoptosis genes had increased mRNA levels; on the contrary, most pro-apoptosis genes had decreased expression levels in due to RXR $\alpha$ deficiency. In male mice, all 4 anti-apoptosis genes in 6 month old mice and 4 out of 5 anti-apoptosis genes in 24 month old mice had increased mRNA levels. On the other hand, 4 out of 7 and 1 out of 1 proapoptosis genes decreased in mRNA expression level, respectively, in 6 and 24 month old male mice. The same trend was also found in female mutant mice. A combination effect of up-regulated anti-apoptosis genes and down-regulated pro-apoptosis genes indicated that RXR $\alpha$ deficient hepatocytes have a more resistant capacity to apoptosis and that hepatocyte RXR $\alpha$ deficiency might have a pro-survival effect.

Among the anti-apoptosis genes, FKBP5 (FK506 binding protein 5) mRNA levels were increased by 4.26- and 2.63fold in 6 month old male and female mice, respectively, due to hepatocyte RXR $\alpha$-deficiency, and the data were confirmed by real-time PCR. FKBP5 is a co-chaperone molecular which interacts with HSP90 (Heat Shock Protein 90) [15]. Its roles include up-regulating the NF-кB pathway and stimulating $\mathrm{Bcl} 2$ transcription. FKBP5 could be up-regulated by androgen, glucocorticoids, and progestin hormones [15]. There is no evidence showing that FKBP5 is directly associated with RXR $\alpha$-mediated signaling. However, RXR $\alpha$ could negatively modulate androgen signaling through binding androgen receptors directly [16]. It is possible that in RXR $\alpha$-deficient hepatocytes, the androgen-mediated signaling could have enhanced activation levels compared with wild-type mice thus leading to higher expression levels of FKBP5.

Another anti-apoptosis gene, USP2 (Ubiquitin Specific Peptidase 2), also showed increased mRNA levels by 3.46and 2.17-fold in 6 month old hepatocyte RXR $\alpha$ deficient male and female mice, respectively. USP2 is a de-ubiquitinase protein and increases $\mathrm{Mdm} 2$ (mouse double minute 2) [17] and FAS (fatty acid synthase) protein stability [18]. Since Mdm2 is responsible for p53 degradation, USP2 could negatively regulate the p53 pathway activity through up-regulation of $\mathrm{Mdm} 2$. In prostate cancer cells, USP2 interacts with and stabilizes FAS, which is often over-expressed in biologically aggressive human tumors. Functional inactivation of USP2 results in decreased FAS protein and enhanced apoptosis in prostate cancer [18]. As with FKBP5, USP2 is also up-regulated by androgen. The similarly elevated expression patterns for FKBP5 and USP2 genes suggest that they are likely regulated by the same mechanism, possibly up-regulation of androgen signaling activity due to RXR $\alpha$ deficiency. In addition, the qRT-PCR results showed that in both genders, the levels of FKBP5 and USP2 mRNA were not increased in 24 month old mutant mice probably due to decreased androgen level in aged mice.

We previously showed that hepatocyte RXR $\alpha$-deficient mice have increased serum cholesterol and triglyceride levels [5], indicating an altered fatty acid metabolism pathway. Our results implied elevated serum triglyceride and cholesterol levels might in part be due to increased activity of FAS because of up-regulation of USP2. Collectively, the changed trends in apoptosis related genes implied that RXR $\alpha$-deficient hepatocytes have an increased resistance to apoptosis. 
Table 3: Fold changes of the mRNA levels of the apoptosis-related genes in male and female hepatocyte RXR $\alpha$-deficient mouse livers.

\begin{tabular}{cccccc}
\hline & 6 month male mice (KO vs. WT) & & 6 month female mice (KO vs. WT) \\
\hline Name & Fold Change & function & Name & Fold Change \\
\hline FKBP5 & 4.26 & anti-apoptosis & FKBP5 & 2.63 & function \\
USP2 & 3.46 & anti-apoptosis & USP2 & 2.17 & anti-apoptosis \\
CFLAR & 2.56 & anti-apoptosis & BCL6 & anti-apoptosis \\
BGN & 2.4 & anti-apoptosis & SERPINA3K & 2.06 & anti-apoptosis \\
BIK & 4.41 & pro-apoptosis & BGN & -2.12 & anti-apoptosis \\
ACVR2B & 3.55 & Pro-apoptosis & & anti-apoptosis \\
BNIP2 & 2.09 & pro-apoptosis & & pro-apoptosis \\
CASP8 & -2.04 & pro-apoptosis & & & \\
EMP2 & -2.7 & pro-apoptosis & & & \\
DUSP6 & -4.26 & pro-apoptosis & & &
\end{tabular}

24 month male mice (KO vs. WT)

24 month female mice (KO vs. WT)

\begin{tabular}{cclccc}
\hline Name & Fold Change & function & Name & Fold Change \\
\hline USP2 & 3.97 & anti-apoptosis & IER3 & 2.23 & Function \\
FKBP5 & 2.81 & anti-apoptosis & BCL6 & anti-apoptosis \\
IER3 & 2.8 & anti-apoptosis & BIK & 2.22 & anti-apoptosis \\
DUSPI & 2.3 & anti-apoptosis & DIABLO & -2.35 & pro-apoptosis \\
BIRC4 & -3.19 & anti-apoptosis & & pro-apoptosis \\
CASPI & 2.83 & pro-apoptosis & & pro-apoptosis \\
UBEIL & 2.36 & pro-apoptosis & & \\
ZBTBI6 & 2.19 & pro-apoptosis & & \\
SOX9 & -2.09 & pro-apoptosis & & \\
CASP8 & -2.27 & pro-apoptosis & & \\
\hline
\end{tabular}

\section{Migration-related genes}

Genes in this group play important roles in cell migration and angiogenesis and are associated with metastasis, a key feature of malignant cancer cells. Generally, the trends observed in this group were different depending upon age and gender (table 4).

In 6 month male RXR $\alpha$-deficient mice, 22 genes showed significant change in mRNA levels. Among those, 6 out of 6 anti-metastasis genes showed reduction of mRNA levels because of RXR $\alpha$ deficiency. Other genes associated with pro-metastasis showed either up- or down-regulation in mRNA levels due to RXR $\alpha$ deficiency. In female hepatocyte RXR $\alpha$-deficient mice, only 6 migration-related genes showed significant changes in their mRNA level.

At 24 month of age, male and female mutant mice showed 9 and 8 genes with alteration in their mRNA levels, respectively. Thus, in aged mice, the difference in the number of genes with deviated mRNA levels is no longer obvious between genders; in contrast, at 6 month of age, the numbers of genes with altered mRNA levels in male and female mutant mice were 22 and 6, respectively. Another striking observation was that many pro-metastasis genes increased their mRNA levels in aged RXR $\alpha$ defi- cient mice. In contrast, very few anti-metastasis associated genes showed change in mRNA levels in aged mice.

In the group of anti-metastasis associated genes, the levels of CD36, THBS1 (thrombospondin 1), and SERPINE1 (Serpin Peptidase Inhibitor) mRNA decreased by 2.16-, 7.11-, and 23.48-fold, respectively, in 6 month old male mutant mice. Real time PCR results showed that THBS1 and SERPINE1 were down-regulated in mRNA levels by 22.21-, and 11.76-fold, respectively. THBS1 is the receptor for CD36 and a potent angiogenesis inhibitor. Down-regulation of THBS1 has been suggested to increase tumor growth and metastasis by modulating angiogenesis in a variety of tumor types [19]. SERPINE1, also named PAI-1 (plasminogen activator inhibitor-1), has been used in gene therapy for inhibition of melanoma metastasis [20]. There were also some pro-metastasis genes, such as CAV1 (caveolin 1) and FN1 (fibronectin 1), which exhibited increased mRNA levels by 2.86- and 2.67-fold, respectively, in 6 month old male mice. On the contrary, in female hepatocyte RXR $\alpha$-deficient mice, those genes, except CD36, did not show changes in expression levels. These observed expression patterns indicate that RXR $\alpha$ deficiency had a greater impact on metastasis related gene expression in males than in female mice at an earlier stage 
Table 4: Fold changes of the mRNA levels of the metastasis-related genes in male and female hepatocyte RXR $\alpha$-deficient mouse livers.

\begin{tabular}{|c|c|c|c|c|c|}
\hline \multicolumn{3}{|c|}{6 month male mice (KO vs. WT) } & \multicolumn{3}{|c|}{6 month female mice (KO vs. WT) } \\
\hline Name & Fold Change & Function & Name & Fold Change & function \\
\hline ARHGDIB & -2.05 & anti-metastasis & BRMSI & -2.16 & anti-metastasis \\
\hline KRTI8 & -2.15 & anti-metastasis & CD36 & -2.88 & anti-metastasis \\
\hline CD36 & -2.16 & anti-metastasis & MYOIO & 2.63 & pro-metastasis \\
\hline KRTI9 & -2.43 & anti-metastasis & ROCKI & -2.24 & pro-metastasis \\
\hline THBSI & -7.11 & anti-metastasis & CTTN & -2.88 & pro-metastasis \\
\hline SERPINEI & -23.48 & anti-metastasis & ITGA6 & -3.64 & pro-metastasis \\
\hline CAVI & 2.86 & pro-metastasis & & & \\
\hline FNI & 2.67 & pro-metastasis & & & \\
\hline ID2 & 2.65 & pro-metastasis & & & \\
\hline DDEFI & 2.52 & pro-metastasis & & & \\
\hline CLCAI & 2.33 & pro-metastasis & & & \\
\hline SIOOAIO & -2.09 & pro-metastasis & & & \\
\hline ADAMI 2 & -2.12 & pro-metastasis & & & \\
\hline CNN2 & -2.16 & pro-metastasis & & & \\
\hline PLAUR & -2.25 & pro-metastasis & & & \\
\hline PLAT & -2.30 & pro-metastasis & & & \\
\hline NEDD9 & -2.39 & pro-metastasis & & & \\
\hline SPPI & -2.75 & pro-metastasis & & & \\
\hline CTGF & -2.90 & pro-metastasis & & & \\
\hline VIM & -3.25 & pro-metastasis & & & \\
\hline LGALS7 & -3.79 & pro-metastasis & & & \\
\hline EPHA2 & -4.71 & pro-metastasis & & & \\
\hline \multicolumn{3}{|c|}{24 month male mice (KO vs. WT) } & \multicolumn{3}{|c|}{24 month female mice (KO vs. WT) } \\
\hline Name & Fold Change & Function & Name & Fold Change & function \\
\hline TIMP4 & 4.07 & anti-metastasis & DDRI & -2.20 & anti-metastasis \\
\hline ANK3 & 2.07 & anti-metastasis & TFF3 & 11.22 & pro-metastasis \\
\hline LGALS3 & 2.00 & anti-metastasis & ADAMI5 & 3.38 & pro-metastasis \\
\hline TFF3 & 4.05 & pro-metastasis & MMP9 & 2.49 & pro-metastasis \\
\hline CTGF & 2.22 & pro-metastasis & ITGBI & 2.48 & pro-metastasis \\
\hline MTIE & 2.19 & pro-metastasis & CSF2RB & 2.32 & pro-metastasis \\
\hline CAVI & 2.15 & pro-metastasis & FOXMI & 2.13 & pro-metastasis \\
\hline PLAUR & 2.03 & pro-metastasis & MYO6 & -4.70 & pro-metastasis \\
\hline РTP4A3 & -2.55 & pro-metastasis & & & \\
\hline
\end{tabular}

of life. It also suggests that hepatocytes in male mutant mice might have more cell movement ability than wild type hepatocytes.

Our data indicated that RXR $\alpha$-deficient hepatocytes might have more metastasis ability than normal hepatocytes. Male mutant mice at 6 month of age had 22 genes with changed their mRNA levels. All 8 anti-metastasis genes showed decreased mRNA levels. When mice were 24 month old, the up-regulation of mRNA levels in prometastasis related genes became more robust in hepatocyte RXR $\alpha$-deficient mice. It has been revealed that $\mathrm{RXR} \alpha$ ligands could inhibit cell migration through deregulation of matrix metalloproteinase-9 or TIMP-1 production [21].
Down-regulation of THBS1 and SERPINE1 when retinoid signaling is compromised provide another mechanism by which retinoids might have an anti-metastasis role. Our data also suggest that the impact of $\operatorname{RXR} \alpha$ on metastasis is gender and age dependent.

\section{Stress response-related genes}

Several HSP (Heat Shock Protein) genes decreased their mRNA levels due to RXR $\alpha$ deficiency (table 5). At 6 month of age, male and female mutant mice had 6 and 2 HSP genes with decreased mRNA levels, respectively. At 24 month of age, two HSP genes, HSPB1 (heat shock 27 kDa protein 1) and HYOU1 (hypoxia up-regulated 1), showed decreased mRNA levels due to RXR $\alpha$ deficiency. 
Table 5: Fold changes of the mRNA levels of the stress-inducible genes in male and female hepatocyte RXR $\alpha$-deficient mouse livers.

\begin{tabular}{|c|c|c|c|c|c|}
\hline \multicolumn{3}{|c|}{6 month male mice (KO vs. WT) } & \multicolumn{3}{|c|}{6 month female mice (KO vs. WT) } \\
\hline Name & Fold Change & function & Name & Fold Change & function \\
\hline GADD45G & 3.89 & stress inducible & HSPA8 & -2.50 & stress inducible \\
\hline DDIT4 & 2.30 & stress inducible & HSPBI & -4.66 & stress inducible \\
\hline DNAJB4 & -2.39 & stress inducible & & & \\
\hline HSPAIB & -3.42 & stress inducible & & & \\
\hline HSPBI & -3.86 & stress inducible & & & \\
\hline HSPAIA & -10.29 & stress inducible & & & \\
\hline DNAJBI & -11.30 & stress inducible & & & \\
\hline ATF3 & -33.85 & stress inducible & & & \\
\hline \multicolumn{3}{|c|}{24 month male (KO vs. WT) } & \multicolumn{3}{|c|}{24 month female (KO vs. WT) } \\
\hline Name & Fold Change & function & Name & Fold Change & function \\
\hline GADD45G & 2.86 & stress inducible & HIFIA & 2.50 & stress inducible \\
\hline DDIT4 & 2.18 & stress inducible & HIPK2 & -2.22 & stress inducible \\
\hline HSPBI & -2.59 & stress inducible & HSPBI & -2.62 & stress inducible \\
\hline HYOUI & -2.75 & stress inducible & HYOUI & -3.54 & stress inducible \\
\hline
\end{tabular}

The HSP gene family is highly conserved in structure from C. elegans to humans. HSP genes constitute the cellular protection mechanism and can be induced by various physical, chemical, and biological factors. In 6 month old RXR $\alpha$-deficient male mice, DNAJB1 (Dnaj homologue, subfamily B, member 1), HSPB1, HSPA1A (Heat Shock 70 KDa Protein 1A), and HSPA1B (Heat Shock 70 KDa Protein 1B) had reduced mRNA levels by 11.30-, 10.29-, 3.86-, and 3.42-fold, respectively. This coordinated downregulation of the HSP family genes indicated that these genes were regulated by common mechanisms. In 6 month old hepatocyte RXR $\alpha$-deficient female mice, only 2 genes (HSPA8 and HSPB1) had reduced mRNA levels of 2.50- and 4.66-fold, respectively. Thus, hepatocyte RXR $\alpha$ deficiency has a greater impact on HSP gene expression in male than in female mice. In aged mice, there was no gender difference in the expression pattern of the HSP family genes related to RXR $\alpha$ deficiency. HSPB1 and HYOU1 both exhibited decreased mRNA levels in male and female aged mutant mice to a similar extent. HSPB1 mRNA levels were consistently decreased in both male and female mutant mice of both age groups.

Some small HSP genes expression levels such as HSP27 can be up-regulated by RXR/RAR heterodimer in lens [22]; furthermore, RXR ligand 9-cis RA (retinoid acid) increases the HSP gene expression in Jurkat cells [23]. Rocchi, P., et al. suggested that the expression of HSPB1 could be upregulated by androgen ablation [24]. Another report revealed that exogenous androgen treatment could delay stress response by decreasing the expression of HSP70 [25]. Down-regulation of these HSP genes implied that RXR $\alpha$-deficient hepatocytes had a reduced protective abil- ity and might be more susceptible to injury resulted from external stimuli compared with wild type hepatocytes. It is possible that there was increased androgen signaling activity due to RXR $\alpha$ deficiency because RXR $\alpha$ is a negative regulator for the androgen pathway, leading to inhibition of HSP family mRNA expression. Another phenomenon that we observed is that the number of HSP family genes which showed alteration in mRNA levels is higher in male than in female when mice are 6 month old. However, this gap decreased when mice were aged. Since androgen levels are higher in male than in female and decrease during aging, the physiological changes of androgen levels may account for this gender- and age-dependent gene expression pattern. It has been shown that RXR $\alpha$-deficient hepatocytes have a shortened lifespan [6]. Our data implied down-regulation of HSP genes expression in RXR $\alpha$-deficient hepatocytes might result in decreased cell protection ability and consequently render the cells prone to death.

\section{Cell growth regulation-related genes}

Retinoids could arrest cell cycle progression and induce apoptosis in many types of cancer cells through activation of RXRs. RXR $\alpha$ signaling plays critical roles in cell growth and differentiation. In RXR $\alpha$ hepatocyte-deficient mice, many genes associated with cell growth had changes in their mRNA levels. Again, this difference in gene expression pattern is gender and age dependent (table 6).

More genes altered expression patterns in male mutant mice compared with female mutant mice at 6 month of age (both in numbers and fold). For example, Jun, Fos, and Myc mRNA levels decreased by 2.87-, 5.74-, and 7.76fold, respectively, in male mutant mice; on the other 
Table 6: Fold changes of the mRNA levels of the cell growth-related genes in male and female hepatocyte RXR $\alpha$-deficient mouse livers.

\begin{tabular}{|c|c|c|c|c|c|}
\hline \multicolumn{3}{|c|}{6 month male mice (KO vs. WT) } & \multicolumn{3}{|c|}{6 month female mice (KO vs. WT) } \\
\hline Name & Fold Change & function & Name & Fold Change & function \\
\hline LCN2 & 6.37 & oncogene & LCN2 & 4.57 & oncogene \\
\hline PIMI & 4.10 & oncogene & GFRAI & 2.15 & oncogene \\
\hline MAFB & 4.07 & oncogene & PTTGI & -4 & oncogene \\
\hline ERBB3 & 2.05 & oncogene & PROMI & -5.61 & oncogene \\
\hline REL & -2.34 & oncogene & PLAGLI & -2.09 & suppressor \\
\hline MDM2) & -2.47 & oncogene & KLF6 & -2.19 & suppressor \\
\hline JUN & -2.87 & oncogene & wWox & -2.2 & suppressor \\
\hline RHOB & -2.93 & oncogene & CAPG & -2.23 & suppressor \\
\hline PTTGI & -2.95 & oncogene & KLF4 & -2.32 & suppressor \\
\hline RHOC & -3.06 & oncogene & GJAI & -2.91 & suppressor \\
\hline ROSI & -3.14 & oncogene & GSN & -3.18 & suppressor \\
\hline SERTADI & -3.7 & oncogene & PEG3 & -5.96 & suppressor \\
\hline PML & -3.9 & oncogene & TPMI & -11.95 & suppressor \\
\hline ETS2 & -4.97 & oncogene & & & \\
\hline FOS & -5.74 & oncogene & & & \\
\hline MYC & -7.7 & oncogene & & & \\
\hline SOCS3 & 3.85 & suppressor & & & \\
\hline MADILI & 3.73 & suppressor & & & \\
\hline TCF2I & 2.82 & suppressor & & & \\
\hline CEBPD & 2.77 & suppressor & & & \\
\hline SSBP2 & 2.27 & suppressor & & & \\
\hline NDRGI & 2.19 & suppressor & & & \\
\hline HMGNI & 2.1 & suppressor & & & \\
\hline SLC22A 18 & 2.08 & suppressor & & & \\
\hline AIMI & 2.05 & suppressor & & & \\
\hline KSRI & 2.04 & suppressor & & & \\
\hline CDKNIA & -2.32 & suppressor & & & \\
\hline MBL2 & -2.34 & suppressor & & & \\
\hline GSN & -2.42 & suppressor & & & \\
\hline TPMI & -2.46 & suppressor & & & \\
\hline PSRCI & -2.78 & suppressor & & & \\
\hline GPX3 & -2.8 & suppressor & & & \\
\hline PERP & -3.42 & suppressor & & & \\
\hline MSXI & -3.5 & suppressor & & & \\
\hline MBDI & -4.15 & suppressor & & & \\
\hline BTG2 & -5.26 & suppressor & & & \\
\hline KLF6 & -5.67 & suppressor & & & \\
\hline EGR2 & -6.03 & suppressor & & & \\
\hline EGRI & -15.88 & suppressor & & & \\
\hline \multicolumn{3}{|c|}{24 month male mice (KO vs. WT) } & \multicolumn{3}{|c|}{24 month female mice (KO vs. WT) } \\
\hline Name & Fold Change & function & Name & Fold Change & function \\
\hline JUN & 2.29 & oncogene & LIMKI & 4.9 & oncogene \\
\hline PTTGI & 2.25 & oncogene & ROSI & 4.51 & oncogene \\
\hline PLK3 & 3.91 & suppressor & CCNDI & 4.44 & oncogene \\
\hline BLNK & 2.13 & suppressor & APC & 2.87 & oncogene \\
\hline GPX3 & 2.11 & suppressor & MAFB & 2.57 & oncogene \\
\hline BEXLI & 2.08 & suppressor & MYBL2 & 2.54 & oncogene \\
\hline WWOX & -2.00 & suppressor & $\mathrm{SH} 2 \mathrm{~B} 2$ & 2.25 & oncogene \\
\hline PEG3 & -2.2 & suppressor & PDGFB & 2.23 & oncogene \\
\hline WISPI & -3.48 & suppressor & E2F3 & 2.08 & oncogene \\
\hline \multirow[t]{3}{*}{ PERP } & -4.03 & suppressor & FLT4 & 2.02 & oncogene \\
\hline & & & MYCLI & -2.08 & oncogene \\
\hline & & & $\mathrm{GPCl}$ & -2.09 & oncogene \\
\hline
\end{tabular}


Table 6: Fold changes of the mRNA levels of the cell growth-related genes in male and female hepatocyte RXR $\alpha$-deficient mouse livers. (Continued)

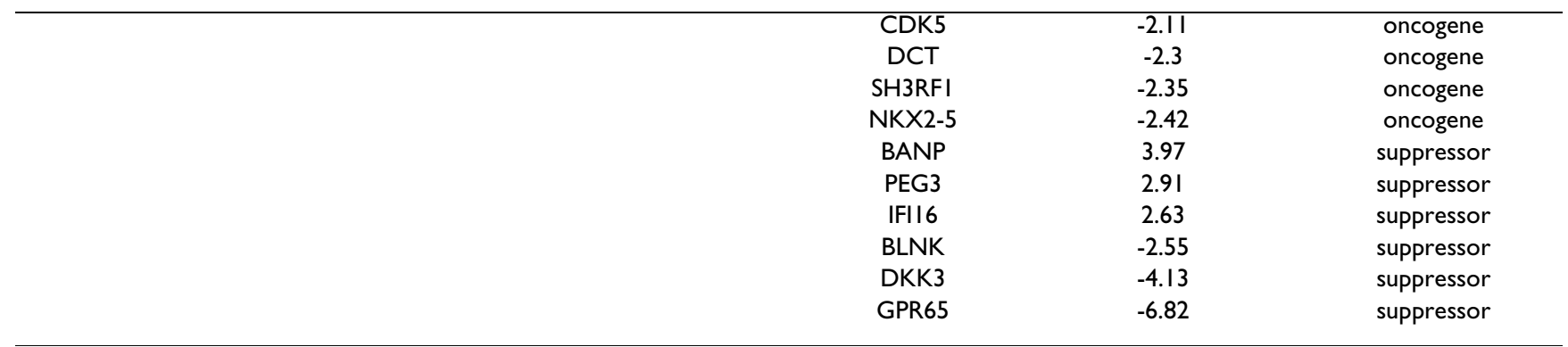

hand, tumor suppressor genes such as KLF6 (Kruppel-like factor 6), EGR2, and EGR1 (early growth response 2 and 1) were down-regulated by 5.67-, 6.03-, and 15.88-fold, respectively. KLF6 inhibits cell proliferation through upregulation of p21 expression in liver cells [26]. EGR1 and 2 , early transcription factors, increase apoptosis in tumor cells. The down-regulation of oncogenes indicated that in RXR $\alpha$-deficient hepatocytes, the cell growth activity was compromised, providing another reason for the observed shortened cell lifespan due to RXR $\alpha$ deficiency. The same trend observed in tumor suppressor genes suggested that negative regulation of cell cycle was also impaired. For example, p21 is up-regulated by activation of RXR/RAR dimer in HepG2 cells [27]. Consistently, our data showed the decrease of p21 mRNA levels by 2.32 folds in 6 month old male mutant mice. BTG2 (B-cell translocation gene 2), a downstream effector of the p53 pathway [28], also had a 5.26 fold reduction. Several genes belonging to the p53 pathway had altered mRNA levels and lead to compromised p53 pathway activity. The impairment of the p53 pathway and other negative regulators implied that due to RXR $\alpha$ deficiency hepatocytes would surpass the cell cycle barrier more easily and be transformed into malignant cells. In female mutant mice, the mRNA levels of the above mentioned genes were not changed. One obvious trend the data points to is that all negative regulator genes for cell cycle transition decreased their mRNA levels in female mutant mice. It also implies cell cycle checkpoint machinery is impaired in female mutant mice.

Contrary to 6 month old female mutant mice, 24 month old female mutant mice had more genes with modified mRNA levels than did the male mutant mice of the same age group (22 vs. 10). In addition, no obvious trends in gene expression patterns were noted. Oncogenes and tumor suppressor genes were up or down regulated randomly.

The impact of RXR-mediated pathways on cell growth is very complex. It can be tissue- or cell type-specific. The activation of RXR/CAR, RXR/PXR, and RXR/PPAR $\alpha$ pathways could induce hepatomegaly [29-31]. On the contrary, RXR/RAR or RXR/VDR pathways inhibit tumor cell growth $[1,32]$. It is likely that the complexity of changes seen in gene expression profiles reflect the net results of combined proliferative and anti-proliferative effects due to hepatocyte RXR $\alpha$ deficiency.

Our data implied that in matured livers, RXR $\alpha$ deficiency has more impact on cell growth-related gene expression levels in males than in females; but in aged liver, female mice have more changes in cell growth-related gene expression patterns than do male mice.

\section{Immune response-related genes}

Immune response-related genes also had significant changes in mRNA level due to RXR $\alpha$ deficiency (table 7). At 6 month of age, male and female mutant mice had 15 and 4 genes with modified mRNA levels, respectively; however, at 24 month of age, there were 10 and 24 genes with change expression levels in male and female mice, respectively. In aged mice, the number of genes with altered mRNA levels increased significantly in female mutant mice. Another striking change in aged mice was that most of the immune response-related genes ( 8 out of 10 in males and 18 out of 24 in females) increased in mRNA levels. Such expression trends were not found in 6 month old mice, implying increased inflammation status might occur in both genders at old age due to RXR $\alpha$ deficiency.

The RXR/PPAR dimer attenuates the inflammation response in colon [32]. RXR and PPAR agonists decrease TNF $\alpha$ (tumor necrosis factor $\alpha$ ) and IL-1 $\beta$ (interleukin $1 \beta$ ) mRNA levels. In liver tissue, the acute response to external stimulus was associated with a down-regulation of RXR $\alpha$ expression [33]. Lipopolysaccharide (LPS) induces a rapid, dose-dependent decrease in $\operatorname{RXR} \alpha, \beta$, and $\gamma$ proteins in hamster liver [33]. Alcohol induced pro-inflammation gene expression (TNF $\alpha$, IL6, and IL1 $\beta$ ) is enhanced due to hepatocyte RXR $\alpha$ deficiency [34]. These observations implied an inverse correlation between inflammation and $\mathrm{RXR} \alpha$ signaling. These findings indicate that $\mathrm{RXR} \alpha$ deficiency increases inflammation response to stimulus, which might be due to deregulation of a panel of immune-related genes. Furthermore, the impact of RXR $\alpha$ 
Table 7: Fold changes of the mRNA levels of the immune response genes in male and female hepatocyte RXR $\alpha$-deficient mouse livers.

\begin{tabular}{|c|c|c|c|c|c|}
\hline \multicolumn{3}{|c|}{6 month male mice (KO vs. WT) } & \multicolumn{3}{|c|}{6 month female mice (KO vs. WT) } \\
\hline Name & Fold Change & function & Name & Fold Change & function \\
\hline DSCRI & -5.50 & immune response & CD9 & -2.2 & immune response \\
\hline CXCLIO & -4.47 & immune response & CD24 & -4.2 & immune response \\
\hline IKBKB & -3.31 & immune response & HLA-G & -2.38 & immune response \\
\hline LIFR & -3.25 & immune response & FSTLI & -2.14 & immune response \\
\hline IGHGI & -2.53 & immune response & & & \\
\hline SI00A9 & -2.39 & immune response & \multicolumn{3}{|c|}{24 month female mice (KO vs. WT) } \\
\hline IGHM & -2.17 & immune response & Name & Fold Change & function \\
\hline MME & -2.17 & immune response & B2M & -2.47 & immune response \\
\hline FCGR2B & -2.14 & immune response & IL7 & -2.38 & immune response \\
\hline $\mathrm{CXXC5}$ & 2.01 & immune response & ILI7RD & -2.28 & immune response \\
\hline CCLI9 & 2.04 & immune response & LIFR & -2.07 & immune response \\
\hline TAXIBPI & 2.13 & immune response & HLA-G & -2.03 & immune response \\
\hline SAA4 & 2.18 & immune response & NFATC2 & -2.03 & immune response \\
\hline $\mathrm{CXCL2}$ & 3.39 & immune response & CDI4 & 2.02 & immune response \\
\hline \multirow[t]{5}{*}{ HLA-G } & 3.76 & immune response & CD48 & 2.02 & immune response \\
\hline & & & FCERIG & 2.05 & immune response \\
\hline & & & HLA-E & 2.05 & immune response \\
\hline & & & NFATC4 & 2.06 & immune response \\
\hline & & & ILIRN & 2.14 & immune response \\
\hline \multicolumn{3}{|c|}{24 month male mice (KO vs. WT) } & IGHM & 2.23 & immune response \\
\hline Name & Fold Change & function & CD24 & 2.38 & immune response \\
\hline ILI3RAI & -2.57 & immune response & SI00A9 & 2.4 & immune response \\
\hline HLA-G & -2.33 & immune response & NFE2 & 2.42 & immune response \\
\hline DSCRI & 2.01 & immune response & ILI2RBI & 2.46 & immune response \\
\hline CCL7 & 2.03 & immune response & LIF & 2.6 & immune response \\
\hline NTRK3 & 2.23 & immune response & ILIA & 2.76 & immune response \\
\hline NTRK 2 & 2.29 & immune response & ILI6 & 2.83 & immune response \\
\hline CXCL3 & 2.47 & immune response & SLPI & 2.97 & immune response \\
\hline CLEC2D & 4.41 & immune response & CD55 & 3 & immune response \\
\hline CXCLIO & 5.92 & immune response & MARCO & 3.24 & immune response \\
\hline IGH-IA & 8.93 & immune response & MEF2C & 5.03 & immune response \\
\hline
\end{tabular}

deficiency on immune response genes was more obvious in aged than in young mice.

\section{The impact of hepatocyte $R X R \alpha$-deficiency on the} expression of gender-dependent genes

The expression of many hepatic genes are gender dependent [35]. However, the findings might vary depending upon the age and strain of mice studied. The susceptibility of night blindness and xerophthalmia, the most common symptoms of vitamin A deficiency, are also gender dependent; the incidence is higher in males than females [36]. Thus, we studied the impact of RXR $\alpha$ deficiency on the expression of hepatic cancer-related genes that have a gender-dependent expression pattern. Our data showed

Table 8: Number of cancer-related genes that showed gender-dependent (male vs. female) expression pattern in 6- and 24-month old wild type and hepatocyte $R X R \alpha$-deficient mice

\begin{tabular}{cccccc}
\hline & Wild Type specific (A) & RXR $\alpha$ deficiency specific (B) & Overlap (C) & Wild type total (A+C) & $R \times R \alpha$ deficiency total (B+C) \\
\hline 6-month & 240 & 111 & 89 & 329 & 200 \\
$24-$ month & 76 & 116 & 51 & 127 & 167 \\
\hline
\end{tabular}


Table 9: Ten gender-dependent genes, which have the greatest fold changes, in wild type and hepatocyte $R X R \alpha$-deficient 6month old mice.

\begin{tabular}{cccc}
\hline \multicolumn{2}{c}{ Male vs. Female in WT } & \multicolumn{2}{c}{ Male vs. Female in KO } \\
\hline Genes in A & Folds & Genes in B & Folds \\
\hline DNAJBI & 26.62 & ILIRI & 31.10 \\
AFP & 22.52 & SOCS3 & 10.63 \\
ATF3 & 19.34 & FMN2 & 8.57 \\
SERPINEI & 14.99 & CXCLI4 & 6.95 \\
HSPAIA & 10.99 & CAVI & 6.00 \\
& & & \\
PROMI & -8.55 & WEEI & -4.98 \\
CLCAI & -9.09 & ID4 & -5.50 \\
DSCRILI & -9.17 & PML & -6.25 \\
CYP2CI9 & -13.83 & GASI & -8.93 \\
PEG3 & -16.00 & PDZRN3 & -10.94 \\
\hline
\end{tabular}

that the numbers of gender-dependent genes in 6 month old wild type and hepatocyte RXR $\alpha$-deficient mice are 329 and 200, respectively. When the mice were aged, the number of gender-dependent genes in wild type mice dropped significantly (127), but not so much in mutant mice (167) suggesting aging narrowed the gender gap more in wild type mice than in the mutant mice (table 8). There were common gender-dependent genes in both wild type and hepatocyte RXR $\alpha$-deficient mice. The names of the genes that showed the greatest fold changes and those genes had the greatest fold changes due to RXR $\alpha$ deficiency at 6-month old age are listed (tables 9 and 10). Also at 24-month old, those genes showed the greatest fold change and genes had the greatest fold changes due to hepatocyte RXR $\alpha$ deficiency are listed in Tables 11 and 12. Surprisingly, the gender-dependent hepatic gene expression was also age-dependent as there was no overlap between the two age groups. Our data indicate that RXR $\alpha$ deficiency affects gender-dependent hepatic gene expression and that this effect is age-dependent.

\section{Conclusion}

Collectively, RXR $\alpha$ deficiency could lead to significant changes in expression levels of cancer-associated genes in a gender- and age-dependent manner. Overall, there is increased resistance for apoptosis; increased cell migration activity; impaired cell protection ability; compromised cell cycle checkpoint machinery, and elevated inflammatory status. These changes may reflect the deregulation of multiple pathways in liver owing to RXR $\alpha$ deficiency. Although 24 month old hepatocyte RXR $\alpha$ deficient mice do not develop spontaneous liver cancer, our data implied that hepatocyte RXR $\alpha$-deficient mice might be more susceptible to cancer development, and this increased risk might be gender- and age-dependent manner.

The current study provides a database of cancer-related hepatic genes that might contribute to a difference in liver cancer incidence between genders as well as due to aging or retinoid signaling deregulation. The limitation of this study is that the role of those genes associated with liver cancer remains to be characterized in actual liver cancer models, which will be done in our future study.

\section{Methods \\ Animals}

Animal protocols were approved by the institutional animal use committee of the University of Kansas Medical Center, Kansas City. Male and Female C57BL/6J wild type and RXR $\alpha$ knock out mice were weaned at 28 days, housed individually, given free access to water, and randomly assigned to study groups. Four groups of mice were used to determine the effects on gene expression profile at two ages in both male and female mice. Each group had 3 mice. At particular time points after birth, 6 month (mature) and 24 month (aged) mice were sacrificed by cervical dislocation, and the livers were rapidly excised

Table 10: Top ten gender-dependent cancer-related genes that have the greatest fold change due to hepatocyte RXR $\alpha$ deficiency.

\begin{tabular}{cccc}
\hline & & Male vs. Female (6 month old mice) & \\
\hline Genes in C & Folds (WT) & Folds (KO) & Fold change due to RXR $\alpha$ deficiency \\
\hline MYC & 33.49 & 5.52 & 5.07 \\
CDKNIA & 27.04 & 5.03 & 3.13 \\
CYPI7AI & -32.26 & -10.32 & 3.03 \\
BTG2 & 10.53 & 3.48 & 2.87 \\
HSPAIB & 23.96 & 8.35 & 0.46 \\
ABCBI & -8.77 & -19.23 & 0.45 \\
B4GALNTI & -2.73 & -6.06 & 0.41 \\
PTP4A3 & -4.33 & -10.67 & 0.34 \\
CXCL2 & 2.89 & 8.40 & 0.33 \\
HSPBI & 6.40 & 19.48 &
\end{tabular}


Table I I: Ten gender-dependent genes, which have the greatest fold changes, in wild type and hepatocyte $R X R \alpha$-deficient 24month old mice.

\begin{tabular}{cccc}
\hline \multicolumn{2}{c}{ Male vs. Female in WT } & \multicolumn{2}{c}{ Male vs. Female in KO } \\
\hline Genes in A & Folds & Genes in B & Folds \\
\hline SNCA & 6.22 & CLEC2D & 45.07 \\
SOX9 & 4.86 & ASNS & 27.09 \\
CYP26AI & 4.70 & RBICCI & 11.82 \\
ABCG2 & 4.01 & FMN2 & 9.39 \\
BCL6 & 3.46 & RAMP2 & 4.13 \\
& & & \\
PRLR & -3.98 & ITGA6 & -5.78 \\
CXCLI0 & -5.29 & DCT & -6.10 \\
CD79B & -7.09 & PSCDBP & -6.33 \\
IL7 & -9.71 & PLA2G7 & -6.45 \\
DSCRILI & -10.94 & CD55 & -13.57 \\
\hline
\end{tabular}

and flash frozen in liquid nitrogen. No signs of pathology were detected in any of the animals used.

\section{RNA Isolation and Preparation for Microarray Hybridization}

Total RNA was isolated from frozen mouse livers using Trizol Reagent (Invitrogen Corporation, Carlsbad, CA) and further purified using the RNeasy Mini Kit (Qiagen Inc., Valencia, CA). Total RNA was quantified by UV spectrophotometry and its integrity and quality were assessed on RNA 6000 Nano LabChips with the Bioanalyzer 2100 (Agilent Technologies, Palo Alto, CA). Total RNA was reverse transcribed into cDNA using reverse transcription kit provided by Invitrogen Company. Synthesis and purification of double-stranded cDNA were conducted as described in the Expression Analysis Technical Manual (Affymetrix, Santa Clara, CA). Biotin-labeled cRNA was synthesized from the purified cDNA using the BioArray High Yield Transcript Labeling Kit according to the manu- facturer's specifications (ENZO Life Sciences, Farmingdale, NY). Labeled cRNA was purified using the GeneChip Sample Cleanup Module, quantified by UV spectrophotometry and assessed for quality with the Bioanalyzer 2100 . Twenty $\mu \mathrm{g}$ purified cRNA was fragmented and $15 \mu \mathrm{g}$ fragmented cRNA was hybridized to Affymetrix Mouse Genome 430A 2.0 Array GeneChips (Affymetrix, Santa Clara, CA) according to the Expression Analysis Technical Manual. Washing and staining of the hybridized arrays were carried out using the Fluidics Station 400 and arrays were subsequently scanned with the Hewlett Packard GeneArray Scanner.

\section{Microarray Data Analysis}

Affymetrix scan data (.cel files) were imported into Rosetta Resolver for analysis (Rosetta Biosoftware, Seattle, WA). Following intrachip normalization and background correction, individual replicates were combined into single "ratio experiments" by an error-weighted method using the control group as a baseline. An agglomerative hierarchical clustering algorithm utilizing an errorweighted Euclidian distance measure was performed on the ratio experiments to identify active genes. Transcripts were defined as active if they increased or decreased by greater than 2.0-fold. The microarray data from this work was submitted to the ArrayExpress database and the accession number is E-MEXP-1711.

\section{Confirmation of mRNA level changes by quantitative real- time PCR}

The synthesized cDNA was diluted 20 fold by water. All primers and probes were designed based on nucleotide sequences in Genbank using the Primer Express software (PE Applied Biosystems). PCR reaction efficiency was calculated for each primer pair using with five dilution points of the calibrator sample to validate primers and probes. The PCR product covered at least two exons according to introns-exons organisation of selected genes.

Table 12: Top ten gender-dependent cancer-related genes that have the greatest fold change due to hepatocyte RXR $\alpha$ deficiency.

\begin{tabular}{cccc}
\hline & & Male vs. Female & \\
\hline Genes in C & Folds (WT) & Folds (KO) & Fold change due to RXR $\alpha$ deficiency \\
\hline SERPINBI & -9.62 & -2.41 & 3.99 \\
CYP3A4 & -43.86 & -11.68 & 3.75 \\
PROMI & -6.14 & -3.13 & 1.96 \\
CD74 & -3.86 & -2.02 & 1.91 \\
OSGINI & 4.45 & 2.34 & 1.91 \\
CAVI & 2.14 & 3.74 & 0.57 \\
IGHM & -2.28 & -5.68 & 0.40 \\
SI00A8 & -6.80 & -21.10 & 0.32 \\
MTIE & -2.03 & -6.62 & 0.31 \\
SI00A9 & -6.10 & -31.35 & 0.19
\end{tabular}


Each real-time PCR reaction consisted of $1 \times$ PCR Master Mix (PE Applied Biosystems), $0.5 \mu \mathrm{M}$ forward and reverse primers and $1 \mathrm{uM}$ corresponding probe. Final volume is $20 \mu \mathrm{l}$. Reactions were carried out on ABI PRISM 7900 real time PCR system (PE Applied Biosystems) for 40 cycles $\left(95^{\circ} \mathrm{C}\right.$ for $15 \mathrm{~s}, 60^{\circ} \mathrm{C}$ for $\left.1 \mathrm{~min}\right)$. The expression fold change for each gene was calculated using the $\mathrm{Ct}$ method and $\beta$-actin was used as an internal control.

\section{Competing interests}

The authors declare that they have no competing interests.

\section{Authors' contributions}

Professor WYJ designed and supervised the project; HL bred mice and extracted hepatic RNA; GL and LML conducted the microarray experiment; GML performed the data analysis and wrote the manuscript. All authors read and approved the final manuscript.

\section{Acknowledgements}

This work is supported by NIH grants CA53596, AA I 208I, AA I 4 I47, and COBRE grant P20 RR021940. We thank Ms. Barbara Brede for proofreading of this manuscript.

\section{References}

I. Altucci L, Leibowitz MD, Ogilvie KM, de Lera AR, Gronemeyer $H$ : RAR and RXR modulation in cancer and metabolic disease. Nature reviews 2007, 6( I 0):793-8I0

2. Kanamori T, Shimizu M, Okuno M, Matsushima-Nishiwaki R, Tsurumi $\mathrm{H}$, Kojima S, Moriwaki H: Synergistic growth inhibition by acyclic retinoid and vitamin $\mathrm{K2}$ in human hepatocellular carcinoma cells. Cancer science 2007, 98(3):43I-437.

3. Moriwaki H, Shimizu M, Okuno M, Nishiwaki-Matsushima R: Chemoprevention of liver carcinogenesis with retinoids: Basic and clinical aspects. Hepatol Res 2007, 37 SuppI 2:S299-302.

4. Wan YJ, Cai Y, Lungo W, Fu P, Locker J, French S, Sucov HM: Peroxisome proliferator-activated receptor alpha-mediated pathways are altered in hepatocyte-specific retinoid $X$ receptor alpha-deficient mice. The Journal of biological chemistry 2000, 275(36):28285-28290.

5. Wan YJ, An D, Cai Y, Repa Jj, Hung-Po Chen T, Flores M, Postic C, Magnuson MA, Chen J, Chien KR, French S, Mangelsdorf DJ, Sucov HM: Hepatocyte-specific mutation establishes retinoid $\mathbf{X}$ receptor alpha as a heterodimeric integrator of multiple physiological processes in the liver. Molecular and cellular biology 2000, 20(12):4436-4444.

6. Imai T, Jiang M, Kastner P, Chambon P, Metzger D: Selective ablation of retinoid $X$ receptor alpha in hepatocytes impairs their lifespan and regenerative capacity. Proceedings of the National Academy of Sciences of the United States of America 200I, 98(8):458I-4586

7. Cai Y, Dai T, Ao Y, Konishi T, Chuang KH, Lue Y, Chang C, Wan YJ: Cytochrome $\mathbf{P 4 5 0}$ genes are differentially expressed in female and male hepatocyte retinoid $X$ receptor alpha-deficient mice. Endocrinology 2003, | 44(6):23।I-23।8.

8. Ye P, Wang Z], Zhang XJ, Zhao YL: Age-related decrease in expression of peroxisome proliferator-activated receptor alpha and its effects on development of dyslipidemia. Chinese medical journal 2005, I I 8( I 3): 1093-1098.

9. Feart C, Pallet V, Boucheron C, Higueret D, Alfos S, Letenneur L, Dartigues JF, Higueret $P$ : Aging affects the retinoic acid and the triiodothyronine nuclear receptor mRNA expression in human peripheral blood mononuclear cells. European journal of endocrinology / European Federation of Endocrine Societies 2005 , 152(3):449-458.

10. Frick KM, Burlingame LA, Arters JA, Berger-Sweeney J: Reference memory, anxiety and estrous cyclicity in C57BL/6NIA mice are affected by age and sex. Neuroscience 2000, 95(I):293-307.
II. Cao SX, Dhahbi JM, Mote PL, Spindler SR: Genomic profiling of short- and long-term caloric restriction effects in the liver of aging mice. Proceedings of the National Academy of Sciences of the United States of America 200I, 98(19): 10630-10635.

12. Hanahan D, Weinberg RA: The hallmarks of cancer. Cell 2000, I 00(I):57-70.

13. Karin M, Lawrence T, Nizet V: Innate immunity gone awry: linking microbial infections to chronic inflammation and cancer. Cell 2006, I 24(4):823-835.

14. Harkin DP, Bean JM, Miklos D, Song YH, Truong VB, Englert C, Christians FC, Ellisen LW, Maheswaran S, Oliner JD, Haber DA: Induction of GADD45 and JNK/SAPK-dependent apoptosis following inducible expression of BRCAI. Cell 1999, 97(5):575-586.

15. Park J, Kim M, Na G, Jeon I, Kwon YK, Kim JH, Youn H, Koo Y: Glucocorticoids modulate NF-kappaB-dependent gene expression by up-regulating FKBP5I expression in Newcastle disease virus-infected chickens. Molecular and cellular endocrinology 2007, 278(I-2):7-I7.

16. Chuang KH, Lee YF, Lin WJ, Chu CY, Altuwaijri S, Wan YJ, Chang C: 9-cis-retinoic acid inhibits androgen receptor activity through activation of retinoid $X$ receptor. Molecular endocrinology (Baltimore, Md 2005, 1 9(5): I 200-1212.

17. Stevenson LF, Sparks A, Allende-Vega N, Xirodimas DP, Lane DP, Saville MK: The deubiquitinating enzyme USP2a regulates the p53 pathway by targeting Mdm2. The EMBO journal 2007, 26(4):976-986.

18. Graner E, Tang D, Rossi S, Baron A, Migita T, Weinstein LJ, Lechpammer M, Huesken D, Zimmermann J, Signoretti S, Loda M: The isopeptidase USP2a regulates the stability of fatty acid synthase in prostate cancer. Cancer cell 2004, 5(3):253-26I.

19. Hawighorst T, Oura H, Streit M, Janes L, Nguyen L, Brown LF, Oliver G, Jackson DG, Detmar M: Thrombospondin-I selectively inhibits early-stage carcinogenesis and angiogenesis but not tumor lymphangiogenesis and lymphatic metastasis in transgenic mice. Oncogene 2002, 2 I(52):7945-7956.

20. Ma D, Gerard RD, Li XY, Alizadeh H, Niederkorn JY: Inhibition of metastasis of intraocular melanomas by adenovirus-mediated gene transfer of plasminogen activator inhibitor type I (PAI-I) in an athymic mouse model. Blood 1997, 90(7):2738-2746

21. Kintscher U, Goetze S, Wakino S, Kim S, Nagpal S, Chandraratna RA, Graf K, Fleck E, Hsueh WA, Law RE: Peroxisome proliferatoractivated receptor and retinoid $X$ receptor ligands inhibit monocyte chemotactic protein-I-directed migration of monocytes. European journal of pharmacology 2000, 40 I (3):259-270.

22. Gopal-Srivastava R, Cvekl A, Piatigorsky J: Involvement of retinoic acid/retinoid receptors in the regulation of murine alphaB. crystallin/small heat shock protein gene expression in the lens. The Journal of biological chemistry 1998, 273(28): |7954-|796|

23. Zhang $\mathrm{H}, \mathrm{Wu} \mathrm{N}$, Shen $\mathrm{Y}$ : [The regulation of vitamin $\mathbf{D} 3$ and 9 cis-retinoic acid and their receptors on human hsp90 beta gene]. Zhongguo yi xue ke xue yuan xue bao 2000, 22(4):322-326.

24. Rocchi P, Beraldi E, Ettinger S, Fazli L, Vessella RL, Nelson C, Gleave $\mathrm{M}$ : Increased Hsp27 after androgen ablation facilitates androgen-independent progression in prostate cancer via signal transducers and activators of transcription 3-mediated suppression of apoptosis. Cancer research 2005, 65(23): I I 083-I I 093.

25. Tetzlaff J, Tanzer L, Jones KJ: Exogenous androgen treatment delays the stress response following hamster facial nerve injury. Journal of neuroendocrinology 2007, 19(5):383-389.

26. Narla G, Kremer-Tal S, Matsumoto N, Zhao X, Yao S, Kelley K, Tarocchi M, Friedman SL: In vivo regulation of p2I by the Kruppellike factor 6 tumor-suppressor gene in mouse liver and human hepatocellular carcinoma. Oncogene 2007, 26(30):4428-4434.

27. Suzui M, Shimizu M, Masuda M, Lim JT, Yoshimi N, Weinstein IB: Acyclic retinoid activates retinoic acid receptor beta and induces transcriptional activation of p2I(CIPI) in HepG2 human hepatoma cells. Molecular cancer therapeutics 2004, 3(3):309-316

28. Boiko AD, Porteous S, Razorenova OV, Krivokrysenko VI, Williams BR, Gudkov AV: A systematic search for downstream mediators of tumor suppressor function of $\mathrm{p} 53$ reveals a major role of BTG2 in suppression of Ras-induced transformation. Genes \& development 2006, 20(2):236-252. 
29. Yang Q, Nagano T, Shah Y, Cheung C, Ito S, Gonzalez Fj: The PPAR alpha-humanized mouse: a model to investigate species differences in liver toxicity mediated by PPAR alpha. Toxicol Sci 2008, I 01 (I): 132-139.

30. Yamamoto Y, Moore R, Goldsworthy TL, Negishi M, Maronpot RR: The orphan nuclear receptor constitutive active/androstane receptor is essential for liver tumor promotion by phenobarbital in mice. Cancer research 2004, 64(20):7197-7200.

31. Chen $Y$, Tang $Y$, Wang MT, Zeng S, Nie D: Human pregnane $\mathbf{X}$ receptor and resistance to chemotherapy in prostate cancer. Cancer research 2007, 67(21):10361-10367.

32. Desreumaux P, Dubuquoy L, Nutten S, Peuchmaur M, Englaro W, Schoonjans K, Derijard B, Desvergne B, Wahli W, Chambon P, Leibowitz MD, Colombel JF, Auwerx J: Attenuation of colon inflammation through activators of the retinoid $X$ receptor (RXR)/ peroxisome proliferator-activated receptor gamma (PPARgamma) heterodimer. A basis for new therapeutic strategies. The Journal of experimental medicine 200I, 193(7):827-838.

33. Beigneux AP, Moser AH, Shigenaga JK, Grunfeld C, Feingold KR: The acute phase response is associated with retinoid $X$ receptor repression in rodent liver. The Journal of biological chemistry 2000 , 275(2I): 16390-16399.

34. Gyamfi MA, He L, French SW, Damjanov I, Wan YJ: Hepatocyte retinoid $X$ receptor alpha-dependent regulation of lipid homeostasis and inflammatory cytokine expression contributes to alcohol-induced liver injury. The Journal of pharmacology and experimental therapeutics 2008, 324(2):443-453.

35. Holloway MG, Miles GD, Dombkowski AA, Waxman DJ: Liver-specific hepatocyte nuclear factor-4alpha deficiency: greater impact on gene expression in male than in female mouse liver. Molecular endocrinology (Baltimore, Md 2008, 22(5): | 274-I 286.

36. Holvik K, Meyer HE, Sogaard AJ, Haug E, Falch JA: Pakistanis living in Oslo have lower serum 1,25-dihydroxyvitamin $D$ levels but higher serum ionized calcium levels compared with ethnic Norwegians. The Oslo Health Study. BMC endocrine disorders 2007, 7:9.

Publish with Bio Med Central and every scientist can read your work free of charge

"BioMed Central will be the most significant development for disseminating the results of biomedical research in our lifetime. "

Sir Paul Nurse, Cancer Research UK

Your research papers will be:

- available free of charge to the entire biomedical community

- peer reviewed and published immediately upon acceptance

- cited in PubMed and archived on PubMed Central

- yours - you keep the copyright

Submit your manuscript here:

http://www.biomedcentral.com/info/publishing_adv.asp
BiolMedcentral 